\title{
ON SUFFICIENT CONDITIONS FOR HARMONICITY
}

\author{
BY
}

P. C. FENTON

ABSTRACT. Suppose that $u$ is continuous in the plane and that given any complex number $z$ there is a number $\rho=\rho(z)>0$ such that

$$
u(z)=\frac{1}{2 \pi} \int_{0}^{2 \pi} u\left(z+\rho e^{i \theta}\right) d \theta .
$$

The main result is: if $u$ possesses a harmonic majorant and $\rho(z)$ is continuous and satisfies a further condition (which may not be omitted) then $u$ is harmonic. Another result in the same vein is proved.

1. Introduction. It is well known that a continuous function in a given domain is harmonic if at each point $z$ in the domain

$$
u(z)=\frac{1}{2 \pi} \int_{0}^{2 \pi} u\left(z+\rho e^{i \theta}\right) d \theta
$$

for arbitrarily small values of $\rho$. In his book of problems [3] Littlewood asks whether, in the case of a continuous bounded function in the open unit disc, these requirements can be reduced to the hypothesis that (1.1) holds on only one circle at each point. This question remains unanswered. The purpose of the present paper is to investigate the corresponding problem for the plane.

We shall prove

THEOREM 1. Suppose that $u$ is continuous and that, given any point $z$ in the plane, there is a number $\rho=\rho(z)>0$ such that (1.1) holds. If

(i) there is a harmonic function $h(z)$ such that $u(z)<h(z)$ for all $z$;

(ii) $\rho(z)$ is continuous;

(iii) there is a point $z_{0}$ such that $\left\{z:\left|z-z_{0}\right|<\rho(z)\right\}$ is bounded; then $u$ is harmonic. In fact, $u(z)=h(z)+K$ for some constant $K$.

We shall show by an example that Theorem 1 is false if condition (iii) is omitted. The example is actually bounded which suggests that there is no nontrivial global growth condition on $u$ which, together with (ii), implies that $u$ is harmonic.

We shall prove also

Received by the editors December 30, 1977 and, in revised form, May 9, 1978.

AMS (MOS) subject classifications (1970). Primary 31 A05. 
THEOREM 2. Suppose that $u$ is continuous and bounded in the plane and that, given any point $z$ there is a number $\rho=\rho(z)>0$ such that $(1.1)$ holds. If $\rho(z)$ is bounded then $u$ is harmonic i.e. $u$ is identically constant.

The author is indebted to the referee for the following example which shows that (i) of Theorem 1 cannot be dispensed with even when (ii) and (iii) are strengthened to $\rho(z) \equiv 1$. Let $u(x, y)=e^{i \lambda x}$, where $\lambda$ is a complex solution of the equation $J_{0}(z)=1$. (Here $J_{0}$ is the usual Bessel function.) By Weber's formula (see $[4$, p. 343])

$$
\frac{1}{2 \pi} \int_{0}^{2 \pi} u\left(z+e^{i \theta}\right) d \theta=J_{0}(\lambda) u(z)=u(z)
$$

for all $z$, and the same equation holds for $\operatorname{Re} u$; yet $\operatorname{Re} u$ is not harmonic. This example also shows, incidentally, that some boundedness requirement on $u$ is essential in Theorem 2 .

2. Proof of Theorem 1. By considering $u(z)-h(z)$ it is evident that we may assume that $u(z) \leqslant 0$ and $h(z) \equiv 0$. With this simplification let $v_{\xi}(z)$ be the harmonic function in $|z-\zeta|<\rho(\zeta)$ which is the Poisson integral of the boundary values of $u$ and define (cf. [1, pp. 452-453])

$$
V(z)=\sup _{\zeta} v_{\zeta}(z)
$$

where the supremum is extended over those $\zeta$ such that $|\zeta-z|<\rho(\zeta)$. From (1.1) we have $u(z)=v_{z}(z) \leqslant V(z)$ for all $z$. We have also

LEMMA 1. $V(z)$ is subharmonic.

The proof of Lemma 1 requires

LEMMA 2. Suppose that $v_{\xi_{n}}$ is a sequence taken from the harmonic functions of (2.1) and that $a_{n} \rightarrow a_{0}$ is a convergent sequence satisfying $\left|a_{n}-\zeta_{n}\right|<\rho\left(\zeta_{n}\right)$ for all $n$. If

$$
\left|a_{0}-\zeta_{n}\right|-\rho\left(\zeta_{n}\right) \rightarrow 0 \text { as } n \rightarrow \infty
$$

then

$$
\varlimsup_{n \rightarrow \infty} v_{\xi_{n}}\left(a_{n}\right)<u\left(a_{0}\right) \text {. }
$$

Assuming the validity of both Lemmas 1 and 2 for the moment, let us continue with the proof of Theorem 1.

Since $v_{\zeta}(z) \leqslant 0$ for all $\zeta$, from the maximum principle, it follows that $V(z)<0$ and this together with Lemma 1 implies that $V(z) \equiv K$ for some constant $K<0$. Moreover $u(z) \leqslant V(z)=K$ for all $z$ and combining this with condition (iii) of Theorem 1 we deduce that the supremum in (2.1) is actually attained at the point $z_{0}$ of (iii). For suppose that $\zeta_{n}$ is such that

$$
v_{\xi_{n}}\left(z_{0}\right) \rightarrow V\left(z_{0}\right) \text { as } n \rightarrow \infty \text {. }
$$


Since $\left(\zeta_{n}\right)$ is bounded, by (iii), there is a subsequence of $\left(\zeta_{n}\right)$ convergent to some point $\zeta_{0}$; and for this subsequence $\rho\left(\zeta_{n}\right) \rightarrow \rho\left(\zeta_{0}\right)$ since $\rho$ is continuous. It follows that $V\left(z_{0}\right)=v_{\zeta_{0}}\left(z_{0}\right)$ if $\left|z_{0}-\zeta_{0}\right|<\rho\left(\zeta_{0}\right)$ so that in this case the supremum is attained. On the other hand, if $\left|z_{0}-\zeta_{0}\right|=\rho\left(\zeta_{0}\right)$ then we may apply Lemma 2 (with $a_{n}=z_{0}$ for all $n$ ) to obtain $\overline{\lim } v_{\xi_{n}}\left(z_{0}\right) \leqslant u\left(z_{0}\right)$. Combining this with (2.2) we obtain $V\left(z_{0}\right) \leqslant u\left(z_{0}\right)$, and so $V\left(z_{0}\right)=u\left(z_{0}\right)=v_{z_{0}}\left(z_{0}\right)$. In either case, then, $V\left(z_{0}\right)=v_{w}\left(z_{0}\right)=K$, for some $w$ satisfying $\left|z_{0}-w\right|<\rho(w)$. Now $v_{w}(z) \leqslant K$ in $\Delta(w, \rho(w))$ and hence, from the maximum principle, $v_{w}(z) \equiv K$. In particular, for $z$ on $C(w, \rho(w)), u(z)=v_{w}(z)=K$. (We use here the notation $\Delta(a, b), C(a, b)$ to denote respectively the open disc and the circle with centre $a$ and radius $b$.) We fix any $z$ on $C(w, \rho(w))$ and conclude from (1.1) that $u(\zeta)=K$ for $\zeta$ on $C(z, \rho(z))$. As $z$ traverses the circle $C(w, \rho(w))$ the circles $C(z, \rho(z))$ sweep out a neighbourhood $N$ of $C(w, \rho(w))$, since $\rho$ is continuous, and throughout $N u(z)=K$. By repeating the argument at the boundary points of $N$ we may extend the neighbourhood indefinitely, so covering the whole plane. Thus $u(z)=K$ for all $z$ and Theorem 1 is proved.

3. Proof of the lemmas. First the proof of Lemma 2, which is divided into three cases.

(a) $\rho\left(\zeta_{n}\right) \rightarrow 0$ as $n \rightarrow \infty$, in which case the result is obvious.

(b) $\rho\left(\zeta_{n}\right)$ has a bounded subsequence with nonzero limit. In this case the result follows using a standard argument which may be found in [1, p. 453].

(c) $\rho\left(\zeta_{n}\right) \rightarrow \infty$ as $n \rightarrow \infty$. Select a subsequence of $\left(\zeta_{n}\right)$ such that $\arg \left(a_{0}-\zeta_{n}\right)$ tends to a limit as $n \rightarrow \infty$. The circles $C\left(\zeta_{n}, \rho\left(\zeta_{n}\right)\right)$ then determine a half-plane $H$ passing through $a_{0}$ and having $a_{0}-\zeta_{n}$ as normal to the edge in the limit. Given $\varepsilon$ satisfying $0<\varepsilon<1$, let $t>0$ be so small that $\left|u\left(a_{0}\right)-u(z)\right|<\varepsilon$ for all $z$ in $\Delta\left(a_{0}, t\right)$ and let $D$ be the domain which is the intersection of $\Delta\left(a_{0}, t\right)$ with the half-plane containing $H$ which is obtained by shifting $H$ slightly in the direction of the normal to its edge. $D$ is then slightly more than a half-disc centred at $a_{0}$ and the chord $\Gamma$ forming part of its boundary is parallel to the edge of $H$.

For sufficiently large $n$ the part of $C\left(\zeta_{n}, \rho\left(\zeta_{n}\right)\right)$ which lies in $\Delta\left(a_{0}, t\right)$ also lies in $D$. Moreover for these large $n$ we have on $C\left(\zeta_{n}, \rho\left(\zeta_{n}\right)\right) \cap D$

$$
v_{\zeta_{n}}(z)-u\left(a_{0}\right)=u(z)-u\left(a_{0}\right)<\varepsilon
$$

and on $C\left(a_{0}, t\right) \cap \Delta\left(\zeta_{n}, \rho\left(\zeta_{n}\right)\right)$

$$
v_{\xi_{n}}(z)-u\left(a_{0}\right) \leqslant-u\left(a_{0}\right)<1+\left|u\left(a_{0}\right)\right|
$$

since $v_{\xi_{n}}(z)<V(z) \leqslant 0$. Using a well-known harmonic measure argument we construct a harmonic function in $D$ which dominates $v_{\xi_{n}}(z)-u\left(a_{0}\right)$ on $D \cap \Delta\left(\zeta_{n}, \rho\left(\zeta_{n}\right)\right)$. Given any $z$ in $D$ let $\theta=\theta(z)$ be the angle subtended by $\Gamma$ at $z$ and let $\theta_{0}$ be the angle subtended by $\Gamma$ at the circular part of the 
boundary of $D$. Then

$$
\omega(z)=\left(1+\left|u\left(a_{0}\right)\right|\right)\left\{\frac{\pi-\theta}{\pi-\theta_{0}}\right\}+\varepsilon\left\{1-\frac{\pi-\theta}{\pi-\theta_{0}}\right\}
$$

is harmonic in $D$ and has boundary values $\varepsilon$ on $\Gamma$ and $1+\left|u\left(a_{0}\right)\right|$ on $\partial D \backslash \Gamma$. It follows from the minimum principle that $\omega(z)>\varepsilon$ on $C\left(\zeta_{n}, \rho\left(\zeta_{n}\right)\right) \cap D$ and hence from (3.1) and (3.2)

$$
\omega(z)>v_{\zeta_{n}}(z)-u\left(a_{0}\right)
$$

for $z$ in $D \cap \Delta\left(\zeta_{n}, \rho\left(\zeta_{n}\right)\right)$. In particular

$$
\omega\left(a_{n}\right) \geqslant v_{\zeta_{n}}\left(a_{n}\right)-u\left(a_{0}\right)
$$

for all large $n$. Further

$$
\lim _{n \rightarrow \infty} \omega\left(a_{n}\right)=\left(1+\left|u\left(a_{0}\right)\right|\right)\left\{\frac{\pi-\theta\left(a_{0}\right)}{\pi-\theta_{0}}\right\}+\varepsilon\left\{1-\frac{\pi-\theta\left(a_{0}\right)}{\pi-\theta_{0}}\right\}
$$

and this approaches $\varepsilon$ as $D$ approaches a half-disc. Since $\varepsilon$ is arbitrary it follows from (3.4) that

$$
\varlimsup_{n \rightarrow \infty} v_{\xi_{n}}\left(a_{n}\right)-u\left(a_{0}\right) \leqslant 0
$$

which proves Lemma 2.

Turning to the proof of Lemma 1 we show first that $V$ is upper semicontinuous. Suppose, on the contrary, that there is a sequence $a_{n} \rightarrow a_{0}$ such that $V\left(a_{n}\right) \rightarrow \lambda>V\left(a_{0}\right)$. Then there is a sequence $\zeta_{n}$ such that

$$
v_{\xi_{n}}\left(a_{n}\right) \rightarrow \lambda>V\left(a_{0}\right) \geqslant u\left(a_{0}\right) \text {. }
$$

It follows from Lemma 2 that there is a neighbourhood $N$ of $a_{0}$, of radius $\varepsilon_{0}$ say, which is contained in $\Delta\left(\zeta_{n}, \rho\left(\zeta_{n}\right)\right)$ for all large $n$.

The harmonic functions $v_{\xi_{n}}(z)$ are nonpositive in $N$ so from Harnack's inequality $[2$, p. 262] there is a constant $\alpha$ independent of $n$ such that for all $z$ in $N^{\prime}=\Delta\left(a_{0}, \frac{1}{2} \varepsilon_{0}\right)$

$$
v_{\zeta_{n}}(z)>\alpha v_{\zeta_{n}}\left(a_{n}\right) \text {. }
$$

Hence $v_{\zeta_{n}}(z)$ is uniformly bounded in $N^{\prime}$ and thus a subsequence converges uniformly in $N^{\prime}$ to a harmonic function $\psi(z)$. We have $\psi\left(a_{0}\right)=\lambda$ and hence, for arbitrarily large $n, v_{\xi_{n}}\left(a_{0}\right)>V\left(a_{0}\right)$, a contradiction. Hence $V$ is upper semicontinuous.

To show that $V$ is subharmonic we consider two cases. If, for a given $\zeta$, $V(\zeta)=u(\zeta)$ then for all small positive $r$

$$
\begin{aligned}
V(\zeta) & =u(\zeta)=v_{\zeta}(\zeta)=\frac{1}{2 \pi} \int_{0}^{2 \pi} v_{\zeta}\left(\zeta+r e^{i \theta}\right) d \theta \\
& <\frac{1}{2 \pi} \int_{0}^{2 \pi} V\left(\zeta+r e^{i \theta}\right) d \theta .
\end{aligned}
$$


Otherwise $V(\zeta)>u(\zeta)$ in which case there is a sequence $v_{\zeta_{n}}$ such that $v_{\zeta_{n}}(\zeta) \rightarrow V(\zeta)$. Also, from Lemma 2 , there is a neighbourhood $\Delta\left(\zeta, \varepsilon_{1}\right)$ contained in $\Delta\left(\zeta_{n}, \rho\left(\zeta_{n}\right)\right)$ for all large $n$. Arguing as before we see that $v_{\zeta_{n}}(z)$ is uniformly bounded in $\Delta\left(\zeta, \frac{1}{2} \varepsilon_{1}\right)$ and so a subsequence converges uniformly in $\Delta\left(\zeta, \frac{1}{2} \varepsilon_{1}\right)$ to a harmonic function $\Phi(z) \leqslant V(z)$. Since $\Phi(\zeta)=V(\zeta)$ we obtain for all small positive $r$

$$
V(\zeta)=\frac{1}{2 \pi} \int_{0}^{2 \pi} \Phi\left(\zeta+r e^{i \theta}\right) d \theta<\frac{1}{2 \pi} \int_{0}^{2 \pi} V\left(\zeta+r e^{i \theta}\right) d \theta .
$$

This completes the proof of Lemma 1 .

4. Proof of Theorem 2. As before we define $V(z)$ by (2.1) and conclude that $V$ is subharmonic. Quite similarly we may define

$$
W(z)=\inf v_{\zeta}(z),
$$

the infimum extended over those $\zeta$ such that $|\zeta-z|<\rho(\zeta)$; and $W$ is superharmonic. Since $V$ and $W$ are bounded in the plane they are constant, say $V(z)=K_{1}, W(z)=K_{2}$. Further $K_{1} \geqslant u(z) \geqslant K_{2}$ for all $z$. We prove

LEMMA 3. There are points $z_{1}, z_{2}$ in the plane at which $u\left(z_{1}\right)=K_{1}, u\left(z_{2}\right)=$ $K_{2}$.

Let $\zeta$ be any fixed point in the plane. Since $V(\zeta)=K_{1}$ there is a sequence $v_{\zeta_{n}}$ such that $v_{\zeta_{n}}(\zeta) \rightarrow V(\zeta)$. Since $\rho\left(\zeta_{n}\right)$ is bounded the sequence $\zeta_{n}$ is bounded and hence there is a subsequence of $\zeta_{n}$ such that $\zeta_{n} \rightarrow \zeta_{0}, \rho\left(\zeta_{n}\right) \rightarrow \rho_{0}$. If $\rho_{0}=0$ then obviously $\zeta_{0}=\zeta$ and $K_{1}=V(\zeta)=u(\zeta)$, and $z_{1}=\zeta_{0}$ is a suitable choice. Similarly if $\left|\zeta-\zeta_{0}\right|=\rho_{0}$, for then again $V(\zeta)=u(\zeta)$, from Lemma 2 . Otherwise $\rho_{0}>0$ and $\left|\zeta-\zeta_{0}\right|<\rho_{0}$ and it is easily verified in this case that $v_{\zeta_{n}}(\zeta) \rightarrow \Psi(\zeta)$, where $\Psi$ is the harmonic function in $\Delta\left(\zeta_{0}, \rho_{0}\right)$ which is the Poisson integral of the boundary values of $u$. Hence $\Psi(\zeta)=K_{1}$ and, since $u(z)<K_{1}$ everywhere, we have $\Psi(z) \equiv K_{1}$ and $u(z)=K_{1}$ on $C\left(\zeta_{0}, \rho_{0}\right)$, the circle centred at $\zeta_{0}$ of radius $\rho_{0}$. This proves half the lemma and the other half is proved similarly.

We aim to show that $K_{1}=K_{2}$ and to this end we suppose $K_{1} \neq K_{2}$ and deduce a contradiction. Define

$$
S^{\prime}=\left\{z: u(z)=K_{1}\right\}, \quad S^{\prime \prime}=\left\{z: u(z)=K_{2}\right\},
$$

both nonempty closed sets. Choose $R$ so large that $\Delta_{0}=\overline{\Delta(0, R)}$ contains points of both $S^{\prime}$ and $S^{\prime \prime}$ and let $d>0$ be the distance of $\Delta_{0} \cap S^{\prime}$ from $\Delta_{0} \cap S^{\prime \prime}$. Then there are points $z_{1}^{\prime} \in \Delta_{0} \cap S^{\prime}, z_{1}^{\prime \prime} \in \Delta_{0} \cap S^{\prime \prime}$, such that $\mid z_{1}^{\prime \prime}-$ $z_{1}^{\prime} \mid=d$. We have $u(z)=K_{1}$ on $C\left(z_{1}^{\prime}, \rho\left(z_{1}^{\prime}\right)\right)$ and $u(z)=K_{2}$ on $C\left(z_{1}^{\prime \prime}, \rho\left(z_{1}^{\prime \prime}\right)\right)$. From the definition of $d$ then, one of $\Delta\left(z_{1}^{\prime}, \rho\left(z_{1}^{\prime}\right)\right)$ and $\Delta\left(z_{1}^{\prime \prime}, \rho\left(z_{1}^{\prime \prime}\right)\right)$ must contain the other, say

$$
\Delta\left(z_{1}^{\prime}, \rho\left(z_{1}^{\prime}\right)\right) \subset \Delta\left(z_{1}^{\prime \prime}, \rho\left(z_{1}^{\prime \prime}\right)\right)
$$


Let $z_{2}^{\prime}$ be the point in $S^{\prime} \cap \Delta\left(z_{1}^{\prime \prime}, \rho\left(z_{1}^{\prime \prime}\right)\right)$ which is closest to the boundary of $\Delta\left(z_{1}^{\prime \prime}, \rho\left(z_{1}^{\prime \prime}\right)\right)$. Then $\Delta\left(z_{2}^{\prime}, \rho\left(z_{2}^{\prime}\right)\right)$ must contain $\Delta\left(z_{1}^{\prime \prime}, \rho\left(z_{1}^{\prime \prime}\right)\right)$ and so

$$
\rho\left(z_{2}^{\prime}\right) \geqslant \rho\left(z_{1}^{\prime \prime}\right)+d .
$$

Now take $z_{2}^{\prime \prime}$ to be the point in $S^{\prime \prime} \cap \Delta\left(z_{2}^{\prime}, \rho\left(z_{2}^{\prime}\right)\right)$ closest to the boundary of $\Delta\left(z_{2}^{\prime}, \rho\left(z_{2}^{\prime}\right)\right)$. Then

$$
\left|z_{2}^{\prime \prime}-z_{2}^{\prime}\right| \geqslant\left|z_{1}^{\prime \prime}-z_{2}^{\prime}\right| \geqslant\left|z_{1}^{\prime}-z_{1}^{\prime \prime}\right|=d
$$

and $\Delta\left(z_{2}^{\prime \prime}, \rho\left(z_{2}^{\prime \prime}\right)\right)$ must contain $\Delta\left(z_{2}^{\prime}, \rho\left(z_{2}^{\prime}\right)\right)$ so

$$
\rho\left(z_{2}^{\prime \prime}\right) \geqslant \rho\left(z_{2}^{\prime}\right)+d \geqslant \rho\left(z_{1}^{\prime \prime}\right)+2 d .
$$

The process may be continued indefinitely, with $d$ added to the radius at each stage, which contradicts the boundedness of $\rho(z)$. Thus $K_{1}=K_{2}=u(z)$ for all $z$ and Theorem 2 is proved.

5. An example. Define four sequences inductively as follows. Set $r_{1}=1$, $r_{1}^{\prime}=2, r_{1}^{\prime \prime}=3, r_{1}^{\prime \prime \prime}=4$ and define, for $n>1$,

$$
\begin{gathered}
r_{n+1}=2 r_{n}+r_{n}^{\prime \prime}+2^{n-1}, \quad r_{n}^{\prime}=r_{n}+2^{n-1}, \\
r_{n+1}^{\prime \prime}=3 r_{n}^{\prime \prime}+2 r_{n}+3 \cdot 2^{n-1}, \quad r_{n}^{\prime \prime \prime}=r_{n}^{\prime \prime}+2^{n-1} .
\end{gathered}
$$

We observe the following relationships which are easily verified:

$$
\begin{gathered}
r_{n}<r_{n}^{\prime}<r_{n}^{\prime \prime}<r_{n}^{\prime \prime \prime}<r_{n+1} ; \\
r_{n}+r_{n}^{\prime \prime \prime}=r_{n+1}-r_{n} ; \\
2 r_{n}^{\prime}+r_{n}^{\prime \prime \prime}=r_{n+1}^{\prime} ; \\
r_{n}^{\prime \prime \prime}=r_{n+1}-2 r_{n} ; \\
2 r_{n}^{\prime}+r_{n+1}^{\prime}=r_{n+1}^{\prime \prime}-2\left(r_{n}^{\prime \prime}-r_{n}^{\prime}\right)<r_{n+1}^{\prime \prime} ; \\
2 r_{n}^{\prime \prime \prime}+r_{n+1}^{\prime}=r_{n+1}^{\prime \prime \prime} ; \\
r_{n+1}^{\prime \prime}-r_{n+1}^{\prime}=2 r_{n}^{\prime \prime} .
\end{gathered}
$$

We define $u(z)$ by $u(z)=1$ for $|z| \leqslant r_{1}=1$ and in general

$$
\begin{array}{ll}
u(z)=1-2^{1-n}\left(|z|-r_{n}\right) & \left(r_{n}<|z|<r_{n}^{\prime}\right) ; \\
u(z)=0 & \left(r_{n}^{\prime} \leqslant|z|<r_{n}^{\prime \prime}\right) ; \\
u(z)=2^{1-n}\left(|z|-r_{n}^{\prime \prime}\right) & \left(r_{n}^{\prime \prime}<|z|<r_{n}^{\prime \prime \prime}\right) ; \\
u(z)=1 & \left(r_{n}^{\prime \prime \prime}<|z|<r_{n+1}\right) .
\end{array}
$$

Then $u(z)$ is continuous everywhere. We define $\rho(z)=4+|z|$ for $|z|<r_{1}=$ 1 and in general (leaving aside for the moment questions of existence)

$\rho(z)$ is the smallest number no less than $r_{n}^{\prime \prime \prime}+|z|$ such that

$$
\begin{gathered}
u(z)=\frac{1}{2 \pi} \int_{0}^{2 \pi} u\left(z+\rho(z) e^{i \theta}\right) d \theta \quad\left(r_{n}<|z|<r_{n}^{\prime}\right) . \\
\rho(z)=|z|+r_{n+1}^{\prime}\left(r_{n}^{\prime} \leqslant|z| \leqslant r_{n}^{\prime \prime}\right) .
\end{gathered}
$$


$\rho(z)$ is the smallest number no less than $r_{n+1}^{\prime}+|z|$ at which (5.8) holds $\left(r_{n}^{\prime \prime}<|z|<r_{n}^{\prime \prime \prime}\right)$.

$\rho(z)=r_{n+1}^{\prime \prime \prime}+|z|\left(r_{n}^{\prime \prime \prime} \leqslant|z| \leqslant r_{n+1}\right)$.

We shall show by induction that $u(z)$ has the mean value property on circles of radius $\rho(z)$ and that $\rho(z)$ is continuous. (The reader is cautioned that the accompanying picture of $u(z)$ in the range $r_{n}<|z|<r_{n+1}$ is not drawn to scale.) Suppose this is true for $|z| \leqslant r_{n}$-it is certainly true for $|z|<r_{1}$-and consider first a fixed $z$ satisfying $r_{n} \leqslant|z| \leqslant r_{n}^{\prime}$. We show that $\rho(z)$ exists. For $\zeta$ on $C\left(z,|z|+r_{n}^{\prime \prime \prime}\right)$ we have, from (5.3),

$$
r_{n}^{\prime \prime \prime} \leqslant|\zeta| \leqslant 2|z|+r_{n}^{\prime \prime \prime} \leqslant 2 r_{n}^{\prime}+r_{n}^{\prime \prime \prime}=r_{n+1}^{\prime} \text {, }
$$

so, from the definition of $u$, for $\zeta$ on $C\left(z,|z|+r_{n}^{\prime \prime \prime}\right) u(\zeta)>u\left(2|z|+r_{n}^{\prime \prime \prime}\right)$. Further, from (5.4), $2|z|+r_{n}^{\prime \prime \prime}=r_{n+1}+2\left(|z|-r_{n}\right)$ so

$$
u\left(2|z|+r_{n}^{\prime \prime \prime}\right)=1-2^{-n} \cdot 2\left(|z|-r_{n}\right)=u(z) \text {. }
$$

Hence $u(\zeta)>u(z)$ for $\zeta$ on $C\left(z,|z|+r_{n}^{\prime \prime \prime}\right)$ and thus $u(z)$ is no larger than its mean value on $C\left(z,|z|+r_{n}^{\prime \prime \prime}\right)$. On the other hand, for $\zeta$ on $C\left(z,|z|+r_{n+1}^{\prime}\right)$,

$$
r_{n+1}^{\prime} \leqslant|\zeta| \leqslant 2|z|+r_{n+1}^{\prime} \leqslant 2 r_{n}^{\prime}+r_{n+1}^{\prime}<r_{n+1}^{\prime \prime} \text {, }
$$

from (5.5), and in this range $u(\zeta)=0$. Hence $u(z)$ is no less than its mean value on $C\left(z,|z|+r_{n+1}^{\prime}\right)$. Since $u$ is continuous the mean value property holds on $C(z,|z|+\rho)$ for some $\rho$ satisfying $|z|+r_{n}^{\prime \prime \prime}<\rho<|z|+r_{n+1}^{\prime}$, so $\rho(z)$ is well defined for $r_{n} \leqslant|z| \leqslant r_{n}^{\prime}$.

To show that $\rho(z)$ is continuous for $r_{n} \leqslant|z|<r_{n}^{\prime}$ it is evidently enough to show that $\rho(x)$ is continuous for $r_{n}<x<r_{n}^{\prime}$. With $x_{0}$ fixed and satisfying $r_{n}<x_{0}<r_{n}^{\prime}$ suppose that, with $r_{n}<x<r_{n}^{\prime}$,

$$
\lambda=\lim _{x \rightarrow x_{0}} \rho(x)<\rho\left(x_{0}\right) .
$$

Then $\lambda \geqslant \lim _{x \rightarrow x_{0}}\left(x+r_{n}^{\prime \prime \prime}\right)=x_{0}+r_{n}^{\prime \prime \prime}$ and, by continuity, the mean value of $u$ on $C\left(x_{0}, \lambda\right)$ is $u\left(x_{0}\right)$, which contradicts the definition of $\rho\left(x_{0}\right)$, since $\lambda<\rho\left(x_{0}\right)$. Hence

$$
\lim _{x \rightarrow x_{0}} \rho(x)>\rho\left(x_{0}\right)
$$

Suppose next that, with $r_{n} \leqslant x \leqslant r_{n}^{\prime}$,

$$
\mu=\varlimsup_{x \rightarrow x_{0}} \rho(x)>\rho\left(x_{0}\right) .
$$

It follows that $x_{0}+r_{n}^{\prime \prime} \leqslant \rho\left(x_{0}\right)<\mu<x_{0}+r_{n+1}^{\prime}$ and that the mean value of $u$ on $C\left(x_{0}, \mu\right)$ is $u\left(x_{0}\right)$. However, as the reader will be readily convinced by the diagram, for $x_{0}+r_{n}^{\prime \prime \prime} \leqslant r \leqslant x_{0}+r_{n+1}^{\prime}, u\left(x_{0}+r e^{i \theta}\right)$ is nonincreasing for each fixed $\theta$ and actually decreases for some $\theta$. It follows that 


$$
\begin{aligned}
u\left(x_{0}\right) & =\frac{1}{2 \pi} \int_{0}^{2 \pi} u\left(x_{0}+\mu e^{i \theta}\right) d \theta \\
& <\frac{1}{2 \pi} \int_{0}^{2 \pi} u\left(x_{0}+\rho\left(x_{0}\right) e^{i \theta}\right) d \theta=u\left(x_{0}\right),
\end{aligned}
$$

a contradiction. Hence, with $r_{n}<x \leqslant r_{n}^{\prime}, \overline{\lim }_{x \rightarrow x_{0}} \rho(x)<\rho\left(x_{0}\right)$ and combining this with (5.9) we deduce that $\rho(x)$ is continuous on $\left[r_{n}, r_{n}^{\prime}\right]$, one-sidedly at the endpoints. Since the two definitions of $\rho\left(r_{n}\right)$ agree, $\rho(z)$ is continuous in $|z|<r_{n}^{\prime}$.

$$
\begin{aligned}
& \text { If } r_{n}^{\prime} \leqslant|z| \leqslant r_{n}^{\prime \prime} \text { then, for } \zeta \text { on } C\left(z,|z|+r_{n+1}^{\prime}\right), \\
& \qquad r_{n+1}^{\prime} \leqslant|\zeta| \leqslant 2|z|+r_{n+1}^{\prime} \leqslant 2 r_{n}^{\prime \prime}+r_{n+1}^{\prime}=r_{n+1}^{\prime \prime},
\end{aligned}
$$

from (5.5), and in this range $u(\zeta)=0$. Hence the mean value of $u$ on $C(z, \rho(z))$ is $0=u(z)$. Once again the two definitions of $\rho\left(r_{n}^{\prime}\right)$ agree so $\rho(z)$ is continuous in $|z|<r_{n}^{\prime \prime}$.

We consider next $z$ satisfying $r_{n}^{\prime \prime} \leqslant|z|<r_{n}^{\prime \prime \prime}$, showing that $\rho(z)$ exists. For $\zeta$ on $C\left(z,|z|+r_{n+1}^{\prime}\right)$,

$$
r_{n+1}^{\prime} \leqslant|\zeta| \leqslant 2|z|+r_{n+1}^{\prime} \leqslant 2 r_{n}^{\prime \prime \prime}+r_{n+1}^{\prime}=r_{n+1}^{\prime \prime \prime} \text {, }
$$

from (5.6), and thus, from the definition of $u, u(\zeta)<u\left(2|z|+r_{n+1}^{\prime}\right)$ for $\zeta$ on $C\left(z,|z|+r_{n+1}^{\prime}\right)$. Moreover, from (5.7),

$$
2|z|+r_{n+1}^{\prime}=r_{n+1}^{\prime \prime}+2\left(|z|-r_{n}^{\prime \prime}\right)
$$

so

$$
u\left(2|z|+r_{n+1}^{\prime}\right)=2^{-n} \cdot 2\left(|z|-r_{n}^{\prime \prime}\right)=u(z) .
$$

We conclude that the mean value of $u$ on $C\left(z,|z|+r_{n+1}^{\prime}\right)$ is no more than $u(z)$. On the other hand, for $\zeta$ on $C\left(z,|z|+r_{n+1}^{\prime \prime \prime}\right)$,

$$
\begin{aligned}
r_{n+1}^{\prime \prime \prime} & <|\zeta|<2|z|+r_{n+1}^{\prime \prime \prime}<2 r_{n}^{\prime \prime \prime}+r_{n+1}^{\prime \prime \prime}=2 r_{n}^{\prime \prime}+r_{n+1}^{\prime \prime}+2^{n} \\
& <2 r_{n+1}+r_{n+1}^{\prime \prime}+2^{n}=r_{n+2},
\end{aligned}
$$

and in this range $u(\zeta)=1$. Hence the mean value of $u$ on $C\left(z,|z|+r_{n+1}^{\prime \prime \prime}\right)$ is $1>u(z)$. Since $u$ is continuous the mean value property holds at $z$ on $C(z, \rho)$ for some $\rho$ satisfying $|z|+r_{n+1}^{\prime}<\rho<|z|+r_{n+1}^{\prime \prime} \cdot \rho(z)$ is thus well defined for $r_{n}^{\prime \prime}<|z|<r_{n}^{\prime \prime \prime}$ and an argument similar to the one used in the range $r_{n}<|z|$ $<r_{n}^{\prime}$ may be used again to show that $\rho(z)$ is continuous in $r_{n}^{\prime \prime}<|z|<r_{n}^{\prime \prime \prime}$. The two definitions of $\rho\left(r_{n}^{\prime \prime}\right)$ agree and so $\rho(z)$ is continuous for $|z|<r_{n}^{\prime \prime \prime}$.

For $r_{n}^{\prime \prime \prime}<|z|<r_{n+1}$ we have, for $\zeta$ on $C\left(z,|z|+r_{n+1}^{\prime \prime \prime}\right)$,

$$
r_{n+1}^{\prime \prime \prime} \leqslant|\zeta| \leqslant 2|z|+r_{n+1}^{\prime \prime \prime}<2 r_{n+1}+r_{n+1}^{\prime \prime \prime}=r_{n+2} \text {, }
$$

from (5.4), and in this range $u(\zeta)=1$. Hence the mean value of $u$ on $C(z, \rho(z))$ is $1=u(z)$. The two definitions of $\rho\left(r_{n}^{\prime \prime}\right)$ agree and $\rho(z)$ is thus continuous in $|z|<r_{n+1}$. By induction, then, $\rho(z)$ is continuous for all $z$, which completes the analysis of the example. 


\section{REFERENCES}

1. P. C. Fenton, Functions having the restricted mean value property, J. London Math. Soc. (2) 14 (1976), 451-458.

2. O. D. Kellogg, Foundations of potential theory, Dover, New York, 1953.

3. J. E. Littlewood, Some problems in real and complex analysis, Heath, Lexington, Mass., 1968.

4. Lawrence Zalcman, Mean values and differential equations, Israel J. Math. 14 (1973), 339-352.

Department of Mathematics, Universtry of Otago, Dunedn, New Zenland 